\title{
Theory of frozars and its observable effects. 2. Complete solution for a collapsing dust star
}

\begin{abstract}
Zahid Zakir
Abstract

The Oppenheimer-Snyder (OS) solution of the Einstein equations for a homogeneous dust star at a parabolic velocity $(\mathrm{k}=0)$, as well as the solution for elliptic velocity $(\mathrm{k}=+1)$, obtained by $\mathrm{O}$. Klein and S. Weinberg by two other methods, describe the collapse in the Schwarzschild coordinates $\mathrm{r}$, t. In the paper a complete solution of the dust star collapse is given by these three methods for all three velocities preserving the homogeneity - parabolic, elliptic and hyperbolic $(\mathrm{k}=0, \pm 1)$. The plots of worldlines, visualizing the internal structure of the star on the hypersurfaces of simultaneity $\mathrm{t}=$ const., are presented. They show that for large but finite $\mathrm{t}$, when the surface freezes asymptotically over the star's gravitational radius, each inner layer also freezes near its asymptote, corresponding to the effective gravitational radius for a given layer. As a result, the collapse of the star leads to the formation of the frozar, a frozen star with a completely frozen internal structure. In the late stages of collapse, when local velocities are close to the light velocity, differences in initial velocities are insignificant and all solutions tend to be parabolic. Therefore, after freezing, the observed effects are similar to those which was studied in the first paper.
\end{abstract}

Keywords: relativistic stars, gravitational collapse, frozen stars, frozars, black holes, horizon, singularity

\section{Content}

Introduction

1. Collapse of a thin dust shell with formation of a hollow frozar ........................................3

1.1. Trajectories of shell's particles in the Schwarzschild field of the shell .......................... 3

1.2. $\quad$ The relationship between the proper time and world time .........................................5

1.3. $\quad$ Metrics and worldlines of test particles inside the shell .......................................... 6

2. Complete solution for a dust star in global coordinates..................................................7

2.1. Tolman solution for dust in local comoving coordinates.............................................. 7

2.2. Transformation of solutions from comoving to static coordinates ................................8

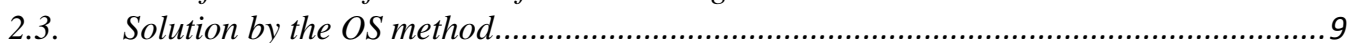

2.4. Solution by the Klein method ...................................................................................... 12

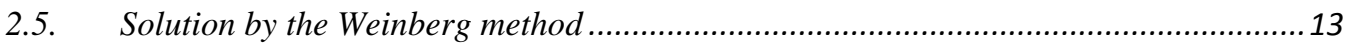

3. The internal structure and evolution of a freezing dust star .....................................15

3.1. Worldlines of layer particles and their asymptotes .............................................. 15

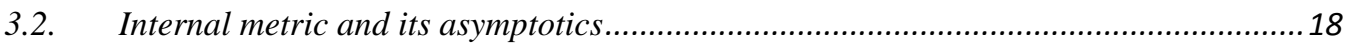

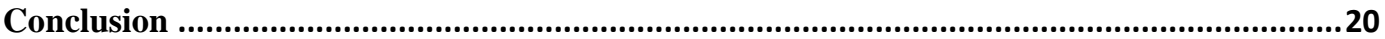

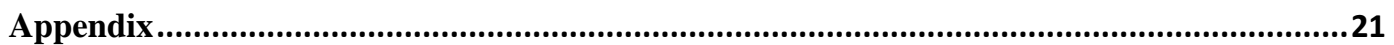

Derivation of an expression for the auxiliary function $y$....................................................... 21

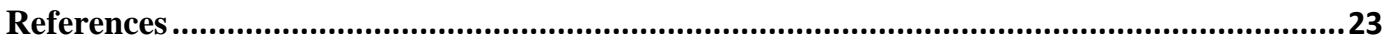

${ }^{1}$ Center for Theoretical Physics and Astrophysics, Tashkent Uzbekistan, zzakir@qgph.org, ORCID 


\section{Introduction}

In the first paper [1], the consequences of the solution [2] of the Einstein equations inside a spherical homogeneous dust star at a parabolic velocity $(k=0)$ of particles in the Schwarzschild coordinates $(t, r, \theta, \varphi)$ was studied. This solution was obtained by Oppenheimer and Snyder (OS) by transformation of the Friedman solution [3] in comoving coordinates (in the form of Tolman [4]) to the Schwarzschild coordinates.

Such a transformation of solutions from the local to the global coordinates is needed both for matching on the surface with the external Schwarzschild solution defined in the global coordinates of the static frame, and for physically correct description of the star's structure by describing the positions of all its layers simultaneously with the surface.

Later, O. Klein [5] and S. Weinberg [6] obtained by two other methods a solution in the Schwarzschild coordinates for the elliptic velocity $(k=+1)$, when the star's particles are at rest at the initial moment. These solutions have not been properly understood long time for methodological and historical reasons.

At first, all this time there was no understanding of the key role of the fact that the local comoving frames do not have a common hypersurface of simultaneity and, therefore, the solutions in these coordinates do not give the positions of the layers simultaneously with the surface, and thus do not determine the instantaneous structure of the entire star.

Secondly, the global solutions are more complicated and their derivations are more cumbersome, moreover, they are difficult to reproduce due to the lack of details of the derivation in the literature. In the OS paper [2], for example, a key auxiliary function $y(R, r)$, main part of their exact solution, has been presented without derivation (the derivation is given in $[7,8])$.

Thirdly, the OS and Klein solutions in the global coordinates $r, t$ were almost never presented in the literature, while the Weinberg's solution, described in his book also without derivation, was considered only as an equivalent form of the simple solution in the local coordinates.

Since the globally defined solutions were misunderstood and practically forgotten, as the result, the physically correct solution of the astrophysical problem, consisting in the definition of the star's structure as a set of simultaneous events, was replaced by a simpler, but a halfway solution, describing the star through non-simultaneous local events.

To overcome this crisis in relativistic astrophysics, a paradigm changing is needed, including three main points. At first, the structure of extended objects, in particular stars, should be described on the global hypersurfaces of simultaneity, where their centers of inertia rests and where symmetries simplify the physical picture. Secondly, the finding of local solutions in suitable coordinates should be considered only as the first stage of the description, and then the global solutions on the hypersurfaces of simultaneity should be constructed from these local solutions. Thirdly, the structure of such objects should be understood as a physical picture following only from the global solutions describing the object at a given moment of world time as a system of simultaneously coexisting particles.

In the paper [7], it is obtained a complete solution by the OS method for a dust star in the case of all three special velocities - parabolic, elliptical and hyperbolic $(k=0, \pm 1)$, including the derivation of the function $y(R, r)$. In the present paper a complete solution 
is given for these three velocities by all three methods - OS, Klein and Weinberg. It is shown that the results of these three methods naturally coincide, since they satisfy the same Einstein equations. The expressions for the asymptotes of the stellar layers for large $t$ are found and the plots of particle's trajectories in the layers of the star are constructed showing the formation of a frozen star or frozar at general relativistic collapse. A systematic presentation of the theory of frozars and its applications will be presented in the book [9].

In Section 1 the evolution of a star's surface in its own gravitational field is described. In Section 2 a solution for a locally homogeneous dust star and complete solutions of the model in the Schwarzschild coordinates are presented. In Section 3 the structure and evolution of a dust star with the formation of frozars are studies and plots of worldlines are presented. In the Appendix the derivation of the function $y(R, r)$ in the OS method is presented.

\section{Collapse of a thin dust shell with formation of a hollow frozar}

\subsection{Trajectories of shell's particles in the Schwarzschild field of the shell}

The space-time interval on the spherical shell and outside it in static coordinates $(t, r, \theta, \varphi)$ in the general case has the form:

$$
d s^{2}=\beta(t) \alpha_{r} d t^{2}-\alpha_{r}^{-1} d r^{2}-r^{2} d \Omega^{2}, \quad r \geq r_{b},
$$

where $\alpha_{r} \equiv 1-r_{g} / r, d \Omega^{2}=d \theta^{2}+\sin ^{2} \theta d \varphi^{2}, r_{g}=2 m G, G$ - gravitational constant and $r_{b}(t)$ is the radius of the circumference of the shell (in the notations of OS [2] and [10]). Outside the shell, the field is static and it is natural to choose $\beta(t)=1$ when $t$ coincides with the proper time of distant observers, and this gives the Schwarzschild metric. In the case of radial incidence, the initial positions of the particles on the shell at the moment

Let the initial radial coordinate of the shell $r=R$ is fixed at $\tau(R, R)=0$, where $\tau(R, r)$ is the proper time. The energy conservation condition then takes the form:

$$
\frac{\alpha_{r}}{1-v_{r}^{2}}=1-k \frac{r_{g}}{R}, \quad k=0, \pm 1
$$

which gives an expression for the local radial velocity:

$$
v_{r}^{2}=\frac{1}{\alpha_{r}^{2}} \frac{d r^{2}}{d t^{2}}=\frac{r_{g}}{r} \frac{1-k r / R}{1-k r_{g} / R} .
$$

Parabolic velocity ( $k=0$ ) corresponds to a fall from rest at $r \rightarrow \infty$, elliptic velocity $(k=+1)$ corresponds to a fall from rest $v_{R}=0$ at $\tau=0$ and $r=R<\infty$, while hyperbolic velocity $(k=-1)$ corresponds to a velocity determined from (3), greater than a parabolic one at $r=R$. At these special velocities, the initially uniform dust particles freely fall radially, remaining be locally homogeneous along the shell.

The proper time of the shell particles is found from (1) and (3):

$$
d \tau^{2}=\alpha_{r} d t^{2}-\alpha_{r}^{-1} d r^{2}=\frac{d r^{2}}{\alpha_{r}}\left(v_{r}^{-2}-1\right)=\frac{r}{r_{g}} \cdot \frac{d r^{2}}{1-k r / R},
$$


which leads to the integral:

$$
\tau(R, r)=\frac{R^{1 / 2}}{r_{g}^{1 / 2}} \int_{r(\tau)}^{R} \frac{d r r^{1 / 2}}{(R-k r)^{1 / 2}} .
$$

Integration gives $\tau(R, r)$, the worldline $r(R, \tau)$ in the implicit form:

$$
\begin{gathered}
\tau(R, r)=\tau_{R}^{(k)}+\frac{R^{3 / 2}}{r_{g}^{1 / 2}}\left(\arccos \bar{h} \frac{r^{1 / 2}}{R^{1 / 2}}+k\left[\frac{r}{R}\left(1-\frac{k r}{R}\right)\right]^{1 / 2}\right), \\
\tau_{R}^{(+1)}=0, \quad \tau_{R}^{(-1)}=\left[2^{1 / 2}-\ln \left(1+2^{1 / 2}\right)\right] R^{3 / 2} / r_{g}^{1 / 2} .
\end{gathered}
$$

Here $\arccos \bar{h}$ means $\arccos$ at $k=1$, and $\operatorname{arccosh}$ at $k=-1$.

Worldline in terms of $t$, i.e. $r(R, t)$, we find from (3):

$$
t=\frac{\left(R-k r_{g}\right)^{1 / 2}}{r_{g}^{1 / 2}} \int_{r(t)}^{R} \frac{d r r^{3 / 2}}{\left(r-r_{g}\right)(R-k r)^{1 / 2}} .
$$

Integration gives:

$$
\begin{aligned}
& t-t_{R}^{(k)}=2 r_{g} \ln \frac{\left[r\left(R-k r_{g}\right)\right]^{1 / 2}+\left[r_{g}(R-k r)\right]^{1 / 2}}{\left[R\left(r-r_{g}\right)\right]^{1 / 2}}+ \\
& {\left[\left(R+2 k r_{g}\right) \arccos \bar{h} \frac{r^{1 / 2}}{R^{1 / 2}}+k[r(R-k r)]^{1 / 2}\right]\left(\frac{R}{r_{g}}-k\right)^{1 / 2} .}
\end{aligned}
$$

To find $t^{\prime}$, an explicit form of $t_{0}(R)$ is required and we will find it from the diagonality condition of the metric in the comoving frame and the condition $\tau^{\prime}=0$ :

$$
t_{R}^{(+1)}=0, \quad t_{R}^{(-1)}=2^{1 / 2}\left(R-2 r_{g}\right)\left(1+R / r_{g}\right)^{1 / 2} .
$$

Together with (9), this gives the desired expressions for worldlines.

The plots of worldlines $r(R, t)$ and $r(R, \tau)$ in Fig. 1 show that both curves are asymptotic, i.e. at $t \rightarrow \infty$ asymptotically approach $r_{g}: r(R, t) \equiv r(R, \tau(R, t)) \rightarrow r_{g}$.
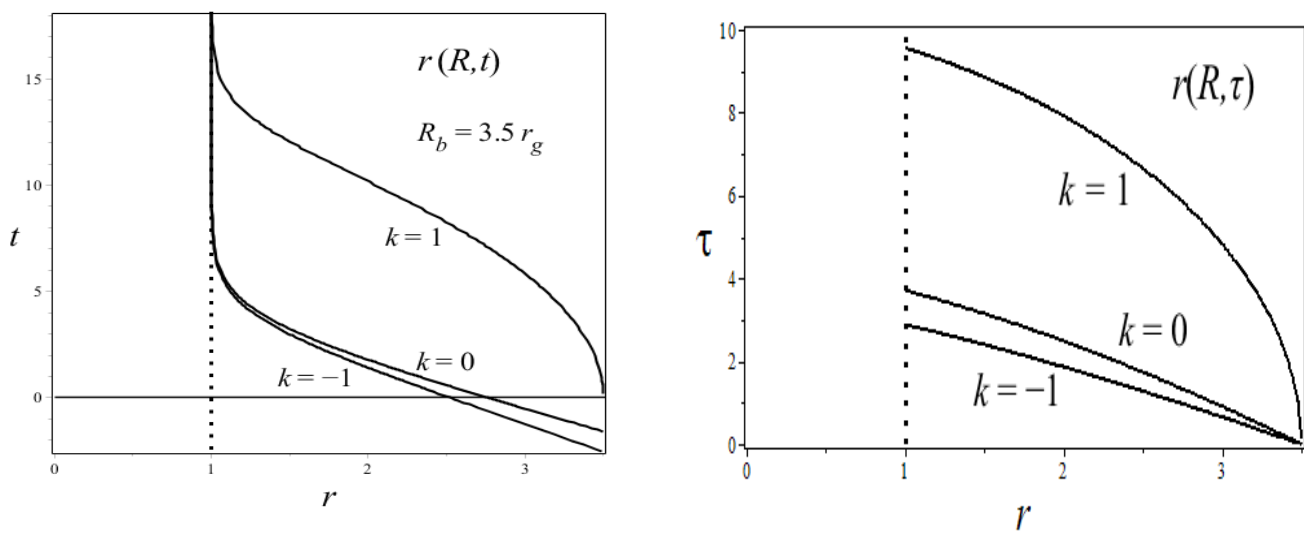

Fig. 1. The worldline $r=r(R, t)=r(R, \tau)$ of a particle on the surface of a star in terms of $t$ (left plot) and $\tau$ (right plot) falling from $R=3.5$ at $\tau=0$ (in units $r_{g}$ ). Events on the worldline $r=r(R, \tau)$ in the right figure are the same events as in the worldline $r=r(R, t)$ in the left figure, i.e. the points of two 
parameterizations of the same events on the same worldline correspond to each other one to one. Thus, the worldlines $r(R, \tau)$ on the right plot are also asymptotic and do not intersect $r_{g}$.

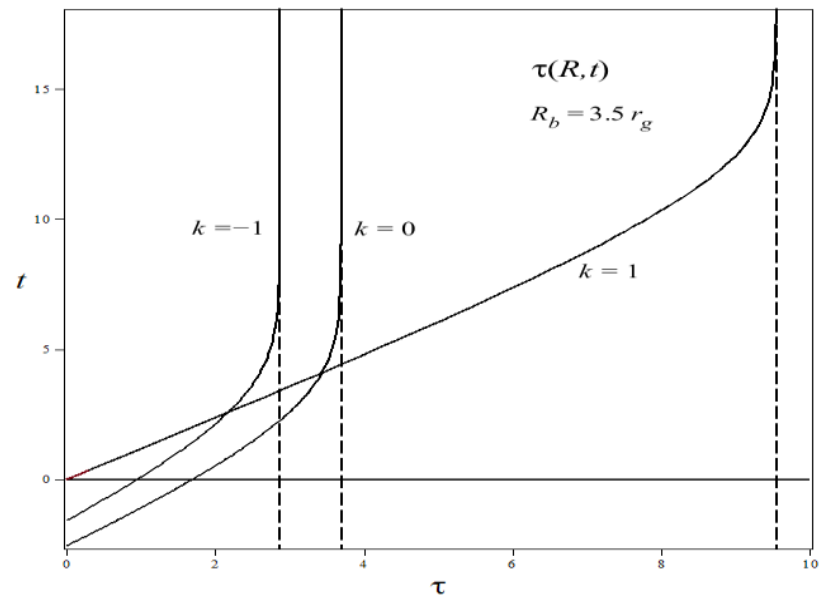

Fig. 2. The relationship $\tau(R, t)$ between proper time $\tau$ and world time $t$ for particles from Fig. 1. The dashed lines are the asymptotes $\tau_{g}$, to which the proper times $\tau$ tend at $t \gg r_{g}$. Values $\tau$ only approach $\tau$, never reaching it at $t<\infty$, and the surface remains outside $t<\infty$ in terms $\tau$ too.

The plot of the relationship between two times $\tau(R, t) \equiv \tau[R, r(R, t)]$ in Fig. 2 shows that at $t \rightarrow \infty$ the proper time $\tau(R, r(t))$ on any layer $R$ tends to an asymptote $\tau_{g}(R)$, the expression for which is presented below.

The time derivative $\dot{r}$ follows directly from (4):

$$
\dot{r}=-\frac{r_{g}^{1 / 2}}{r^{1 / 2}}(1-k r / R)^{1 / 2},
$$

while $r^{\prime}=\partial r / \partial R$ we find from Eqs. (6) - (7) by means the condition $\tau^{\prime}=0$ :

$$
\begin{gathered}
r^{\prime}=\frac{3}{2}-\frac{r}{2 R}+\frac{3}{2}\left(\frac{R}{r}-1\right)^{1 / 2} \arccos \left(r^{1 / 2} / R^{1 / 2}\right), k=+1 \\
r^{\prime}=\frac{3}{2}\left[2^{1 / 2}+\ln \left[\left(1+\frac{r}{R}\right)^{1 / 2}+\frac{r^{1 / 2}}{R^{1 / 2}}\right]\right]\left(\frac{R}{r}+1\right)^{1 / 2}-\frac{3}{2}-\frac{r}{2 R}, \quad k=-1
\end{gathered}
$$

The expression for $\dot{t}$ follows from the Eqs. (3) and (11):

$$
\dot{t}=-\frac{\left[r\left(R-k r_{g}\right)\right]^{1 / 2}}{\left[r_{g}(R-k r)\right]^{1 / 2}} \frac{\dot{r}}{\alpha_{r}}=\frac{\left(1-k r_{g} / R\right)^{1 / 2}}{\alpha_{r}} .
$$

\subsection{The relationship between the proper time and world time}

At $r>r_{g}$ both $t(R, r)$ and $\tau(R, r)$ describe the same event on the same worldline of the same particle (at fixed $\varphi, \theta$ ). Thus, between the moments of proper time $\tau$ and world time $t$ on the shell there is a one-to-one correspondence.

The case of parabolic velocity $k=0$ was studied in detail in the first paper [1] and below the velocity regimes $k= \pm 1$ will be studied. Since the worldline $t(R, r)$ in any velocity regime asymptotically approaches $r_{g}$, never crossing it, the same worldline, 
parameterized through $\tau(R, r)$, also approaches $r_{g}$ only asymptotically (Fig. 1b). The irreversibility of gravitational dilation of $\tau$ w.r.t. $t$ is a fact confirmed by experiments. Here, the kinematical time dilation is also irreversible, as in the twin paradox, due to the accelerated motion of the falling particles relative to the static frame. This can also be seen from the dependence $\tau(R, t)$, which for $k= \pm 1$ can be found only numerically, by calculating $t(R, r)$ and $\tau(R, r)$ at the same $r$. The result is shown in Fig. 2.

At $t \rightarrow \infty$ and $r \rightarrow r_{g}$, the proper time of the falling particle freeze asymptotically, tending to the freezing moment $\tau_{g}^{(k)}$, which manifests itself as an asymptote, to which the proper time tends in the corresponding velocity regime. We find these moments from (6) as:

$$
\begin{gathered}
\tau_{g}^{(0)}=\frac{2}{3 r_{g}^{1 / 2}}\left(R^{3 / 2}-r_{g}^{3 / 2}\right), \\
\tau_{g}^{(-)}=\frac{R^{3 / 2}}{r_{g}^{1 / 2}}\left(2^{1 / 2}-\frac{r_{g}}{R}\left(\frac{R}{r_{g}}+1\right)^{1 / 2}+\ln \left(\frac{r_{g}^{1 / 2}+\left(R+r_{g}\right)^{1 / 2}}{R^{1 / 2}\left(1+2^{1 / 2}\right)}\right)\right), \\
\tau_{g}^{(+)}=r_{g}\left(\frac{R}{r_{g}}-1\right)^{1 / 2}+\frac{R^{3 / 2}}{r_{g}^{1 / 2}} \arccos \frac{r_{g}^{1 / 2}}{R^{1 / 2}} .
\end{gathered}
$$

The worldlines $t(R, r)$ in all three velocity modes approach $r_{g}$ asymptotically, never crossing it and, therefore, the same worldlines parameterized as $\tau(R, r)$ also approach $r_{g}$ asymptotically (Fig. 1b).

\subsection{Metrics and worldlines of test particles inside the shell}

Inside the shell, the same vacuum solution (1) is valid:

$$
d \tau^{2}=\beta(t) \tilde{\alpha}_{r} d t^{2}-\tilde{\alpha}_{r}^{-1} d r^{2}-r^{2} d \Omega^{2}, \quad r<r_{b}(t),
$$

but with $\tilde{\alpha}_{r} \equiv 1-\tilde{r}_{g} / r$. For the lack of singularity in the center, where there is no matter, it must be $\tilde{r}_{g}=0$, which gives $\tilde{\alpha}_{r}=1$. Thus, the space inside the shell is flat.

However, unlike the external region, the field inside the shell, although uniform, is not static, since the choice $\beta(t)=1$ does not allow the external and internal metrics to be matched together. For matching, you must choose $\beta(t)=1-r_{g} / r_{b}(t)$ that at each moment the temporal component of the metric is equal to the value on the shell. As a result, the internal line element takes the form:

$$
d s^{2}=\left[1-r_{g} / r_{b}(t)\right] d t^{2}-d r^{2}-r^{2} d \Omega^{2}, \quad r<r_{b}(t) .
$$

Since only one component of the metric $g_{00}=\beta(t)$ is nonzero, then only one component of the Christoffel symbols is nonvanishing:

$$
\Gamma_{00}^{0}=\frac{1}{2} \frac{\dot{\beta}}{\beta}=\frac{r_{g} \dot{r}_{b}}{2 r_{b}^{2}\left(1-r_{g} / r_{b}\right)}=-\frac{r_{g}^{3 / 2}}{r_{b}^{3 / 2}} \frac{\left(1-k r_{b} / R\right)^{1 / 2}}{2\left(r_{b}-r_{g}\right)} \text {. }
$$


Inside the shell, free test particles move rectilinearly, and for a locally resting system also uniformly $d \mathbf{r} / d \tau=$ const. However, their velocities in terms of world time at $r_{b}(t) \rightarrow r_{g}$ will rapidly decrease:

$$
\mathbf{v}(t)=\frac{d \mathbf{r}}{d t}=\frac{d \mathbf{r}}{d \tau}\left[1-r_{g} / r_{b}(t)\right]^{1 / 2} \rightarrow 0 .
$$

As the shell freezes, each of the test particles inside it also freezes near the point where it was before the shell freezes. Plots of worldlines of such particles are shown in Fig. 3.

\section{Complete solution for a dust star in global coordinates}

\subsection{Tolman solution for dust in local comoving coordinates}

The Tolman solution [4] of the Einstein equations inside the dust star $R \leq R_{b}$, where $R_{b}$ is the initial radial coordinate of the surface is based on the line element in the comoving coordinates:

$$
d s^{2}=c^{2} d \tau^{2}-e^{\lambda(\tau, R)} d R^{2}-r^{2}(R, \tau) d \Omega^{2}
$$

and has the form (see [9]):

$$
e^{\lambda}=\frac{r^{\prime 2}}{1+k f}, \quad \dot{r}^{2}=\frac{F}{r}+k f, 8 \pi G \rho=\frac{F^{\prime}}{r^{\prime} r^{2}} .
$$

Here $1+k f>0, \rho$ is the local energy density of dust matter and two functions $f(R)$, $F(R)$ follow from additional physical conditions. In particular, $F(R)=r_{g}$ at $R \geq R_{b}$, and in the elliptic case $(k=+1) f$ follows from the vanishing of velocities at $r=R$ :

$$
\dot{r}^{2}=\frac{F}{R}+f=0 . \quad f=-\frac{F}{R} .
$$

Local homogeneity, i.e. the coordinate independence of density $\rho=\rho(\tau)$ for the same values of local proper time (for the "same-age" events), is the first such condition. Simplification can be achieved by choosing the initial homogeneous distribution at $\tau=0$ and one of the three special (preserving the homogeneity) velocities with $k=0, \pm 1$. From (23) and (24) then we find:

$$
\dot{r}=-\frac{F^{1 / 2}}{r^{1 / 2}}\left(1-\frac{k r}{R}\right)^{1 / 2}, \quad \tau(R, r)=\int_{r(R, r)}^{R} d r\left(\frac{F}{r}+k f\right)^{-1 / 2} .
$$

The integration gives:

$$
\begin{gathered}
\tau(R, r)=\frac{R^{1 / 2}}{F^{1 / 2}}\left[2^{1 / 2} R-[r(R+r)]^{1 / 2}+R \ln \left(\frac{r^{1 / 2}+(R+r)^{1 / 2}}{R^{1 / 2}\left(1+2^{1 / 2}\right)}\right)\right], k=-1 \\
\tau(R, r)=\frac{R^{1 / 2}}{F^{1 / 2}}\left[[r(R-r)]^{1 / 2}+R \arccos \left(r^{1 / 2} / R^{1 / 2}\right)\right], k=+1 .
\end{gathered}
$$

To find $F$, from (23) and the homogeneity condition, we use the following relations $(\kappa=8 \pi G / 3)$ : 


$$
\begin{gathered}
F^{\prime}=3 \kappa \rho(\tau) r^{2} r^{\prime}=\kappa \rho(\tau)\left(r^{3}\right)^{\prime}, \\
F=\kappa \rho(\tau) r^{3}=\kappa \rho(0) R^{3}=\kappa \rho(0) R_{b}^{3} \frac{R^{3}}{R_{b}^{3}} .
\end{gathered}
$$

Then, taking into account $r_{g}=2 G M=\kappa \rho(0) R_{b}^{3}$ and (24), we arrive at the expressions:

$$
F(R)=r_{g} \frac{R^{3}}{R_{b}^{3}} \equiv r_{g, R}, \quad f(R)=-\frac{r_{g, R}}{R}, \quad \rho(\tau)=\rho(0) \frac{R^{3}}{r^{3}} .
$$

Using $f$ and $F$, we can calculate from (25) not only $\dot{r}$, but $r^{\prime}$ also. At first, from (28)-(30) we obtain:

$$
F^{\prime}=\kappa \rho(\tau) r^{2} r^{\prime}=\kappa \rho(0) R^{3} r^{\prime}=3 r_{g} \frac{R^{3}}{r R_{b}^{3}} r^{\prime},
$$

which, at compared with (30), gives $F^{\prime}=3 R^{2} r_{g} / R_{b}^{3}$, and we find finally:

$$
r^{\prime}=\frac{r}{R}
$$

i.e. $r^{\prime}$ is the same for all three special velocities.

\subsection{Transformation of solutions from comoving to static coordinates}

In the inner region, following OS, we transform the Tolman solution (22) in terms of $R, \tau$ to the solution in terms of $r, t$. This is necessary both for determining the structure of the entire star on the hypersurfaces $t=$ const., and for matching on the surface of internal and exterior metrics.

The general coordinate transformationы:

$$
d x^{i}=\frac{\partial x^{i}}{\partial x^{i^{\prime}}} d x^{i^{\prime}}
$$

at transformation from $d \tau, d R$ to $d t, d r$ take the form:

$$
d t=\dot{t} d \tau+t^{\prime} d R, \quad d r=\dot{r} d \tau+r^{\prime} d R .
$$

The transformed metric $g_{i^{\prime} k^{\prime}}(r, t)$ in the new line element:

$$
g_{i^{\prime} k^{\prime}}=\frac{\partial x^{i}}{\partial x^{i^{\prime}}} \frac{\partial x^{k}}{\partial x^{k^{\prime}}} g_{i k}
$$

is generally non-diagonal. However, it must be diagonal, at least on the surface, in order to be matched with the exterior metric (1). Therefore, the simplest solution in terms of $r, t$ we search for as a diagonal metric.

Notice that the instantaneous structure of the star as an extended object should be determined on the global hypersurfaces of simultaneity coinciding on the surface with the hypersurface of the static frame $t=$ const. This also assumes the presence of a set of synchronized clocks in the interior, showing the same world time $t$ with the surface. All this can naturally be realized with a diagonal metric, but difficult for a non-diagonal metric.

Thus, we proceed from the diagonal line element: 
2. Complete solution for a collapsing dust star

$$
d s^{2}=e^{v(r, t)} d t^{2}-e^{\lambda(r, t)} d r^{2}-r^{2} d^{2} \Omega, \quad r \leq r_{b},
$$

with the matching condition $e^{v\left(r_{b}, t\right)}=e^{-\lambda\left(r_{b}, t\right)}=1-r_{g} / r_{b}$. Substitution (34) into (36) gives:

$$
\begin{aligned}
d s^{2} & =\left(e^{v} \dot{t}^{2}-e^{\lambda} \dot{r}^{2}\right) d \tau^{2}-\left(e^{\lambda} r^{\prime 2}-e^{v} t^{\prime 2}\right) d R^{2}- \\
& -r^{2} d^{2} \Omega+2\left(e^{v} \dot{t} t^{\prime}-e^{\lambda} \dot{r} r^{\prime}\right) d \tau d R .
\end{aligned}
$$

By using (22)-(23), we obtain from (36)-(37):

$$
\begin{gathered}
g_{00}(\tau, R)=e^{v} \dot{t}^{2}-e^{\lambda} \dot{r}^{2}=1, \quad g_{11}(\tau, R)=-\left(e^{\lambda} r^{\prime 2}-e^{v} t^{\prime 2}\right)=-\bar{r}^{\prime 2}, \\
g_{01}(\tau, R)=e^{v} \dot{t} t^{\prime}-e^{\lambda} \dot{r} r^{\prime}=0,
\end{gathered}
$$

where $\bar{r}^{\prime 2}=r^{\prime 2} /(1+k f)$. Eqs. (38) allow us to express $e^{\lambda}$ and $e^{\nu}$ :

$$
e^{\lambda}=\frac{t^{\prime 2}+\dot{t}^{2} \bar{r}^{\prime 2}}{\dot{t}^{2} r^{\prime 2}-t^{\prime 2} \dot{r}^{2}}, \quad e^{v}=\frac{r^{\prime 2}+\bar{r}^{\prime 2} \dot{r}^{2}}{\dot{t}^{2} r^{\prime 2}-t^{\prime 2} \dot{r}^{2}}
$$

The diagonality condition (39), after substituting (40), takes the form:

$$
g_{01}=\frac{\left(r^{\prime 2}+\bar{r}^{\prime 2} \dot{r}^{2}\right) \dot{t} t^{\prime}-\left(t^{\prime 2}+\dot{t}^{2} \bar{r}^{\prime 2}\right) \dot{r} r^{\prime}}{\dot{t}^{2} r^{\prime 2}-t^{\prime 2} \dot{r}^{2}}=0 .
$$

By considering this condition as an equation for $t^{\prime}$ :

$$
t^{\prime 2} \dot{r}-t^{\prime} \dot{t} r^{\prime}\left(1+\frac{\dot{r}^{2}}{1+k f}\right)+\dot{t}^{2} \dot{r} \bar{r}^{\prime 2}=0,
$$

we get two its solutions:

$$
t^{\prime}=\frac{\dot{t} r^{\prime}}{2 \dot{r}}\left(\left[1+\dot{r}^{2} /(1+k f)\right] \pm\left[1-\dot{r}^{2} /(1+k f)\right]\right) .
$$

The choice of the plus sign gives $t^{\prime}=\dot{t} r^{\prime} / \dot{r}$, which is a non-physical solution, since it diverges at $\dot{r} \rightarrow 0$. The choice of the minus sign gives the required diagonality condition:

$$
\frac{t^{\prime}}{\dot{t}}=\frac{\dot{r} r^{\prime}}{1+k f} \text {. }
$$

After exclusion of $t^{\prime}$ by using (44), the metric in (40) simplifies:

$$
e^{\lambda}=\frac{1}{1-F / r}, \quad e^{v}=\frac{1+k f}{\dot{t}^{2}(1-F / r)} .
$$

\subsection{Solution by the OS method}

In the first paper [1], the OS solution for a dust star for a parabolic velocity $(k=0)$ was considered. Here we study the complete solution by the OS method also for elliptic and hyperbolic velocities $(k= \pm 1)$ [5], from which the solution of OS for $k=0$ follows as the limiting case for $r_{g} / R_{b} \ll 1$ or $k \rightarrow 0$.

To determine the worldline $r(t, R)$ in the OC method [2], it is introduced $y_{(k)}(R, r)$ as an auxiliary variable:

$$
t=M_{(k)}\left(y_{(k) b}\right)
$$


where the form of the function $M$ is determined from the matching condition with the exterior metric. The diagonality condition (44) as a result takes the form:

$$
\frac{t^{\prime}}{\dot{t}}=\frac{y_{(k)}^{\prime}}{\dot{y}_{(k)}}=\frac{\dot{r} r^{\prime}}{1-k r_{g, R} / R}=-\frac{\left(r_{g, R} r\right)^{1 / 2}}{R-k r_{g, R}}(1-k r / R)^{1 / 2} .
$$

Solving this equation with respect to $y_{(k)}$ (see Appendix 1), we obtain:

$$
y_{(k)}(R, r)=-\frac{R_{b}}{k r_{g}} \ln \left(\frac{\left(1-k r_{g, R} / R\right)^{1 / 2}(1-k r / R)}{\left(1-k r_{g} / R_{b}\right)^{1 / 2}}\right) .
$$

On the surface $R=R_{b}$, the value $y_{(k)}$ is:

$$
y_{(k)}\left(R_{b}, r\right)=-\left(R_{b} / k r_{g}\right) \ln \left(1-k r_{k} / R_{b}\right) .
$$

Here $r_{k}$ are the values of $r$ leading to the right matching on the surface according (49):

$$
r_{k}=-\left[\exp \left(-k r_{g} y_{(k)} / R_{b}\right)-1\right] R_{b} / k
$$

Inserting in (50) the general expression for $y_{(k)}$ from (48), we obtain $r_{k}(R, r)$ for all layers:

$$
r_{k}(R, r)=\frac{R_{b}}{k}\left(1-\frac{\left(1-k r_{g, R} / R\right)^{1 / 2}(1-k r / R)}{\left(1-k r_{g} / R_{b}\right)^{1 / 2}}\right) .
$$

The function $M_{(k)}\left(y_{(k) b}\right)$ from (46) must coincide with the right-hand side of Eq. (9) for $t(R, r)$. Therefore, substituting $R \rightarrow R_{b}$ in (9), as well as $r \rightarrow r_{k}(R, r)$ in (51), we obtain formulas for $t\left[R_{b}, r_{k}(R, r)\right]$ describing the worldlines $r(R, t)$ (here $r_{ \pm} \equiv r_{ \pm 1}$ ):

$$
\begin{aligned}
& t=2 r_{g} \ln \frac{\left[\left(R_{b}+r_{g}\right) r_{-}\right]^{1 / 2}+\left[r_{g}\left(R_{b}+r_{-}\right)\right]^{1 / 2}}{\left[R_{b}\left(r_{-}-r_{g}\right)\right]^{1 / 2}}-\left[r_{-}\left(r_{-}+R_{b}\right)\right]^{1 / 2}\left(\frac{R_{b}}{r_{g}}+1\right)^{1 / 2}+ \\
& +\left(\frac{R_{b}}{r_{g}}+1\right)^{1 / 2}\left(R_{b}-2 r_{g}\right)\left(2^{1 / 2}+\ln \frac{r_{-}^{1 / 2}+\left(R_{b}+r_{-}\right)^{1 / 2}}{R_{b}^{1 / 2}}\right), \quad k=-1, \\
& t=2 r_{g} \ln \frac{\left[\left(R_{b}-r_{g}\right) r_{+}\right]^{1 / 2}+\left[r_{g}\left(R_{b}-r_{+}\right)\right]^{1 / 2}}{\left[R_{b}\left(r_{-}-r_{g}\right)\right]^{1 / 2}}+\left[r_{+}\left(R_{b}-r_{+}\right)\right]^{1 / 2}\left(\frac{R_{b}}{r_{g}}-1\right)^{1 / 2}+ \\
& +\frac{1}{2}\left(R_{b}+2 r_{g}\right)\left(\frac{R_{b}}{r_{g}}-1\right)^{1 / 2} \arccos \left(\frac{2 r_{+}}{R_{b}}-1\right), \quad k=+1 .
\end{aligned}
$$

Labeling the constants in a fixed layer $R$ as:

$$
a_{ \pm}=\left(1 \mp r_{g} / R_{b}\right)^{1 / 2}, \quad b_{ \pm}=\left(1 \mp r_{g, R} / R\right)^{1 / 2}
$$

and inserting $r_{k}$ from (51) into (52)-(53), we obtain the expressions for $t(R, r)$ describing worldlines $r(R, t)$. For $k=-1$ the result has the form: 


$$
\begin{aligned}
& t=2 r_{g} \ln \frac{a_{+}\left(1+\frac{r}{R}-\frac{a_{-}}{b_{-}}\right)^{1 / 2}+\frac{r_{g}^{1 / 2}}{a_{-}}\left(1+\frac{r}{R}\right)^{1 / 2}}{\left(1+\frac{r}{R}-\frac{a_{-}^{3}}{b_{-}}\right)^{1 / 2}}-R_{b} a_{-}\left[\left(1+\frac{r}{R}\right)\left(1+\frac{r}{R}-\frac{a_{-}}{b_{-}}\right)\right]^{1 / 2} \\
& +\left(R_{b}-2 r_{g}\right) a_{-}\left(2^{1 / 2}+\ln \left[\left(\frac{b_{-}}{a_{-}}\left(1+\frac{r}{R}\right)-1\right)^{1 / 2}+\frac{b_{-}^{1 / 2}}{a_{-}^{1 / 2}}\left(1+\frac{r}{R}\right)^{1 / 2}\right]\right)
\end{aligned}
$$

and for $k=+1$ :

$$
\begin{aligned}
& t=2 r_{g} \ln \frac{\left[a_{+}-b_{+}(1-r / R)\right]^{1 / 2} a_{+}+\left[b_{+}(1-r / R) r_{g} / R_{b}\right]^{1 / 2}}{\left[a_{+}^{3}-b_{+}(1-r / R)\right]^{1 / 2}}+\left(R_{b}+2 r_{g}\right) \times \\
& \times \frac{R_{b}^{1 / 2} a_{+}}{2 r_{g}^{1 / 2}} \arccos \left(1-\frac{2 b_{+}}{a_{+}}\left(1-\frac{r}{R}\right)\right)+\frac{R_{b}^{3 / 2} b_{+}^{1 / 2}}{r_{g}^{1 / 2}}\left(1-\frac{r}{R}\right)\left[a_{+}-b_{+}\left(1-\frac{r}{R}\right)\right]^{1 / 2} .
\end{aligned}
$$

Into the expression for the derivative $\dot{r}_{k}$, following from (51):

$$
\dot{r}_{k}=\dot{r} \frac{R_{b}}{R} \frac{\left(1-k r_{g, R} / R\right)^{1 / 2}}{\left(1-k r_{g} / R_{b}\right)^{1 / 2}},
$$

we substitute $\dot{r}$ from (25) and obtain:

$$
\dot{r}_{k}=-\frac{R_{b}}{R}\left(\frac{r_{g, R}(1-k r / R)\left(1-k r_{g, R} / R\right)}{r\left(1-k r_{g} / R_{b}\right)}\right)^{1 / 2} .
$$

Derivatives $\dot{t}\left(R, r_{k}\right)$, following from (52) and (53), have the form:

$$
\dot{t}_{k}=-\frac{k \dot{r}_{k}}{r_{k}-r_{g}}\left(\frac{r_{k}^{3}\left(R_{b}-k r_{g}\right)}{r_{g}\left(R_{b}-k r_{k}\right)}\right)^{1 / 2} \text {. }
$$

Then, by inserting $\dot{r}_{k}$ from (58), we obtain:

$$
\dot{t}_{k}=\frac{1}{r_{k}-k r_{g}}\left(\frac{r_{k}^{3} R(1-k r / R)\left(1-k r_{g, R} / R\right)}{r\left(R_{b}-k r_{k}\right)}\right)^{1 / 2} .
$$

Let's find the components of metrics. By substituting $F=r_{g, R}$ and $f=-r_{g, R} / R$ into (47), and then replacing $\dot{t}$ with $\dot{t}_{k}$, we obtain:

$$
e^{\lambda}=\frac{1}{1-r_{g, R} / r}, \quad e^{v}=\dot{t}_{k}^{-2} \frac{1-k r_{g, R} / R}{1-r_{g, R} / r} .
$$

The explicit form of $e^{v}$, after substituting $\dot{t}_{k}$ from (60), is: 


$$
e^{v}=\frac{-r / k R}{1-r_{g, R} / r} \cdot \frac{\left(1-\frac{k r}{R}-\frac{\left(1-k r_{g} / R_{b}\right)^{3 / 2}}{\left(1-k r_{g, R} / R\right)^{1 / 2}}\right)^{2}}{\left(1-\frac{k r}{R}-\frac{\left(1-k r_{g} / R_{b}\right)^{1 / 2}}{\left(1-k r_{g, R} / R\right)^{1 / 2}}\right)^{3}} .
$$

In a first approximation, when $r_{g} / R_{b}$ and $r_{g, R} / R$ are small, or in the limit $k \rightarrow 0$, the metric reduces to the parabolic solution of OS [ ].

\subsection{Solution by the Klein method}

The OS method is based on the diagonality condition for the metric on the hypersurface $t=$ const. Another way to go to a diagonal metric is the diagonalization of the general metric and this method was firstly applied to the elliptical case $(k=+1)$ by $\mathrm{O}$. Klein [5].

In this section, we will present a complete solution by the Klein method for all three velocities, which allows us to compare the results with the results of the solution by the OS method.

The Klein's notations are related by the notations of the present paper as:

$$
a \eta=r, a_{0} \eta=R, \quad a_{0} \eta_{0}=R_{b} .
$$

The line element in the comoving coordinates $\tau, \eta$

$$
d s^{2}=d \tau^{2}-a^{2}\left(\frac{d \eta^{2}}{1-k \eta^{2}}+\eta^{2}\right) d \Omega^{2}
$$

was transformed in [5] into a line element in the Schwarzschild coordinates $t, r$. For diagonalization of the transformed line element, the time differential $d t$ was reduced directly to the full differential, and an integrating factor was found from the matching condition.

The resulting line element has a standard form as in (36) with a solution for the metric:

$$
e^{\lambda}=\frac{1}{1-\frac{a_{0}}{a} \eta^{2}}, e^{v}=e^{\lambda} \frac{\left[1-\frac{a_{0}}{k a}\left(1-\frac{\left(1-k \eta_{0}^{2}\right)^{3 / 2}}{\left(1-k \eta^{2}\right)^{1 / 2}}\right)\right]^{2}}{\left[1-\frac{a_{0}}{k a}\left(1-\frac{\left(1-k \eta_{0}^{2}\right)^{1 / 2}}{\left(1-k \eta^{2}\right)^{1 / 2}}\right)\right]^{3}}
$$

The solution for the trajectory takes the form:

$$
t=\frac{2 a_{0}}{k\left(1-k \eta_{0}^{2}\right)^{1 / 2}} \int_{z_{k 0}}^{z_{k}} \frac{z^{4}}{\left(1+k z^{2}\right)^{2}\left(z^{2}-K_{k}^{2}\right)} d z,
$$

where the following notation are introduced: 


$$
K_{k}=\frac{\eta_{0}}{\left(1-k \eta_{0}^{2}\right)^{1 / 2}}, \quad b_{k}=\frac{a_{0}}{k\left(1-k \eta_{0}^{2}\right)^{1 / 2}\left(K_{k}^{2}+k\right)} .
$$

As the result, the Klein elliptic solution $(k=+1)$ has the form [5]:

$$
t=\frac{b_{+} z_{+}}{1+z_{+}^{2}}+\frac{b_{+}}{K_{1}^{2}+1}\left[\left(1+3 K_{+}^{2}\right)\left(\frac{\pi}{2}-\arctan z_{+}\right)+K_{+}^{3} \ln \frac{z_{+}+K_{+}}{z_{+}-K_{+}}\right] .
$$

The hyperbolic solution $(k=-1)$ in (66) has the form:

$$
t=-\frac{b_{-} z_{-}}{z_{-}^{2}-1}+\frac{b_{-}}{K_{k}^{2}-1}\left(K^{3} \ln \frac{z_{-}-K_{-}}{z_{-}+K_{-}}+\frac{1-3 K_{-}^{2}}{2} \ln \frac{z_{-}-1}{z_{-}+1}\right) .
$$

The solutions (65)-(69) obtained by the Klein method can be rewritten in the notations of the present paper (63) by means the relations:

$$
\eta^{2}=\frac{r_{g, R}}{R}=r_{g} \frac{R^{2}}{R_{b}^{3}}, \quad \eta_{0}^{2}=\frac{r_{g}}{R_{b}}, \quad \frac{a}{a_{0}}=\frac{r}{R}, \quad a_{0} \eta_{0}^{3}=r_{g}, \quad a_{0}=\frac{R_{b}}{\eta_{0}}=\frac{R_{b}^{3 / 2}}{r_{g}^{1 / 2}} .
$$

The radial metric in (65) takes the form:

$$
e^{\lambda}=\frac{1}{1-\frac{a_{0}}{a} \eta^{2}}=\frac{1}{1-\frac{R}{r} \frac{r_{g, R}}{R}}=\frac{1}{1-\frac{r_{g, R}}{r}}
$$

and is equal to $e^{\lambda}$ in the solution by the OS method (61). The time component of the metric in (65) takes the form (62).

In the expressions for the worldline (68)-(69), the insert $z_{k}$ from (67) in the form:

$$
z_{k}=\left[\frac{k^{-1}}{1-k r / R}\left(\frac{1-k r_{g} / R_{b}}{1-k r_{g, R} / R}\right)^{1 / 2}-k^{-1}\right]^{1 / 2}
$$

leads to expressions (55)-(56) obtained by the OS method.

As in the OS case, the parabolic solution $(k=0)$ follows from the solutions for $k= \pm 1$ at the limit $k \rightarrow 0$.

Thus, the solutions by the Klein method for $k=0, \pm 1$ are exactly equivalent to the solutions by the OS method.

\subsection{Solution by the Weinberg method}

Another method, where the "integrating factor" technique was also used for the diagonalization of the metric, was presented in Weinberg's book [6] (part 3, section II.9). The notations of this book refer to ours as: 


$$
\begin{aligned}
& \bar{r}=r R(t) \rightarrow r(t, R), \quad t \rightarrow \tau, \quad \bar{t} \rightarrow t, \quad a \rightarrow R_{b}, \quad R(0)=1, R(t)=\frac{r}{R}, \\
& k \rightarrow \tilde{k}=\frac{r_{g}}{a^{3}}, \quad \tilde{k} a^{2}=\frac{r_{g}}{R_{b}}, \quad \tilde{k} r^{2}=\frac{r_{g, R}}{R}=r_{g} \frac{R^{2}}{R_{b}^{3}} .
\end{aligned}
$$

Here, the value $k$ from [6] is noted as $\tilde{k}$, not to be confused with $k$ in the paper.

Line element in the comoving coordinates

$$
d s^{2}=d t^{2}-R^{2}(t)\left(\frac{d r^{2}}{1-\tilde{k} r^{2}}-r^{2} d \Omega^{2}\right)
$$

was transformed to the Schwarzschild coordinates $t, r$. In the new line element:

$$
d s^{2}=B d \bar{t}^{2}-A d \bar{r}^{2}-\bar{r}^{2} d \Omega^{2}
$$

the solution for the metric has the form:

$$
\begin{gathered}
A=\frac{1}{1-\tilde{k} r^{2} / R}, \quad B=\frac{R}{S}\left(\frac{1-\tilde{k} r^{2}}{1-\tilde{k} a^{2}}\right)^{1 / 2} \frac{\left(1-\tilde{k} a^{2} / S\right)^{2}}{1-\tilde{k} r^{2} / R}, \\
S(r, t)=1-\left(\frac{1-\tilde{k} r^{2}}{1-\tilde{k} a^{2}}\right)^{1 / 2}[1-R(t)] .
\end{gathered}
$$

The solution for the worldline is given as the integral:

$$
\bar{t}=\frac{\left(1-k \cdot \tilde{k} a^{2}\right)^{1 / 2}}{\tilde{k}^{1 / 2}} \int_{S(r, t)}^{1} \frac{d x x^{3 / 2}}{\left(x-\tilde{k} a^{2}\right)(1-k x)^{1 / 2}} .
$$

By inserting the notations (73) into (77), we obtain the function $r_{k}$ from the OS method (51):

$$
S(R, r)=\frac{1}{k}\left[1-\left(\frac{1-k r_{g, R} / r}{1-k r_{g} / R_{b}}\right)^{1 / 2}(1-k r / R)\right]=\frac{1}{R_{b}} r_{k}(R, r)
$$

and solution (78) takes the form of solution (8) by the OS method.

The radial metrics $A$ from (76) takes the form

$$
A=\frac{1}{1-\frac{\tilde{k} r^{2}}{R(t)}}=\frac{1}{1-\frac{R}{r} \frac{r_{g, R}}{R}}=\frac{1}{1-\frac{r_{g, R}}{r}},
$$

coinciding with $e^{\lambda}$ in the solution by the OS method (61). The time component of metric $B$ from (76) takes the form

$$
B=\frac{r}{R}\left(\frac{1-r_{g, R} / R}{1-r_{g} / R_{b}}\right)^{1 / 2} \frac{\left(S-r_{g} / R_{b}\right)^{2}}{\left(1-r_{g, R} / r\right) S^{3}}
$$

and after insertion $S$ from (79) appears as equal the $e^{v}$ from (62).

Thus, all three methods, OS [2], Klein [5] and Weinberg [6], lead to the same exact solution of the Einstein equations for the homogeneous dust star. This fact not only means 
the correctness of these methods of solution, but also once again demonstrates the fundamental nature of this solution for the general relativistic collapse of a homogeneous dust star, which does not depend on the methods of derivation.

\section{The internal structure and evolution of a freezing dust star}

\subsection{Worldlines of particles in the layers and their asymptotes}

a) Parabolic velocities $(k=0)$.

The plots of worldlines $r(R, t)$ particles inside a star for the OS parabolic solution were studied in detail the first paper [1] and here we present only the main results. The corresponding plots are shown in Fig. 3 which shows the asymptotes $r_{\infty}^{(0)}(R)$ to which the worldlines of particles in the layers $r(R, t)$ tend at $t \rightarrow \infty$. Asymptotes parallel to the worldline of center are located at almost equal distance, slightly condensing near the surface.

The surface of a star asymptotically approaches its gravitational radius $r_{b}(t) \rightarrow r_{g}$ and at $t \gg r_{g}$, the asymptotics for the surface the form:

$$
\begin{aligned}
& r_{b}(t) \simeq r_{g}\left(1+a e^{-t / r_{g}}\right)>r_{g}, \\
& a \equiv 4 \exp \left(\frac{2 R_{b}^{3 / 2}}{3 r_{g}^{3 / 2}}-\frac{8}{3}\right) .
\end{aligned}
$$

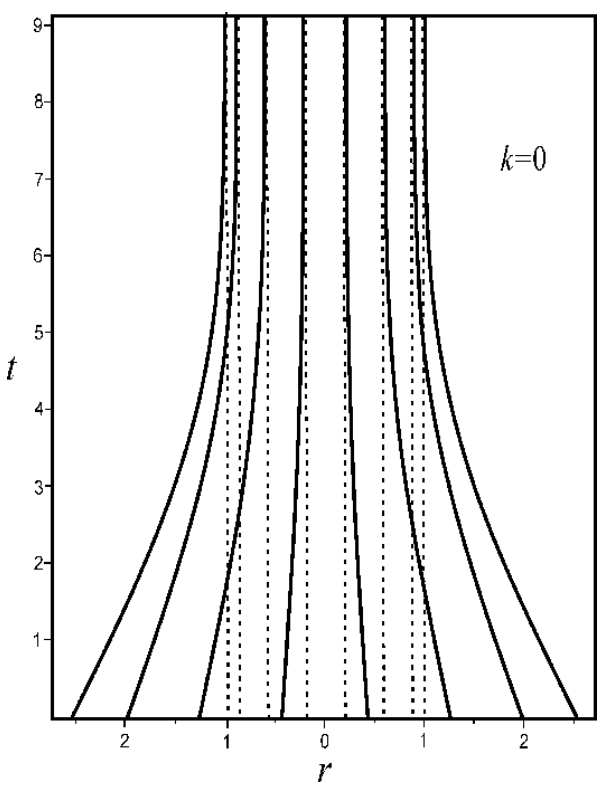

Fig. 3. Worldlines of dust star particles along its diameter according the OS solution at the parabolic velocity $(k=0)$ and $R_{b}=3.5$ at $\tau=0$ (in units $r_{g}$ ) At $t \gg r_{g}$ the surface freezes over $r_{g}$, the inner layers freeze over asymptotes $r_{\infty}(R)$ (dashed lines) given by (84). of

in

the

take

Inside the star at $t \gg r_{g}$ we have $y_{(0)} \rightarrow 1$ and the asymptotics of $r(R, t)$ takes the form:

$$
r(R, t) \simeq R \frac{3 r_{g}}{2 R_{b}}\left(1-\frac{R^{2}}{3 R_{b}^{2}}+a e^{-t / r_{g}}\right) .
$$

This leads to the expression for asymptotes:

$$
r_{\infty}^{(0)}(R)=R \frac{3 r_{g}}{2 R_{b}}\left(1-\frac{R^{2}}{3 R_{b}^{2}}\right)=r_{g, R}+\frac{3 r_{g, R}}{2}\left(\frac{R_{b}^{2}}{R^{2}}-1\right) .
$$

where $r_{\infty}^{(0)}\left(R_{b}\right)=r_{g}$. This shows that $r_{\infty}^{(0)}(R)$ is an "internal" gravitational radius of the layer $R$, which is effectively formed by the gravitational radius $r_{g, R}$ of the matter inside 
the layer, as well as the second term (84), which describes the contribution of time dilation due to external layers.

As follows from (84), the asymptotes of the close to the center inner layers are located almost equidistantly $\sim R$ (Fig. 3), and only layers near the surface become denser due to spatial compressions and subsequent freezing, which is described by a factor $-r_{g} R^{3} / 2 R_{b}^{3}$.

We find the asymptotic behavior of the proper times of particles in the stellar layers by substituting (83) in the expression for $\tau(R, r)[1]$ :

$$
\tau(R, t)=\frac{2 R_{b}^{3 / 2}}{3 r_{g}^{1 / 2}}-\frac{3^{1 / 2} r_{g}}{2^{1 / 2}}\left(1-\frac{R^{2}}{3 R_{b}^{2}}+a e^{-t / r_{g}}\right)^{3 / 2} .
$$

Proper times in each layer freeze at values $\tau_{\infty}^{(0)}(R)$ :

$$
\begin{gathered}
\tau_{\infty}^{(0)}(R)=\frac{2 R_{b}^{3 / 2}}{3 r_{g}^{1 / 2}}-\frac{3^{1 / 2} r_{g}}{2^{1 / 2}}\left(1-\frac{R^{2}}{3 R_{b}^{2}}\right)^{3 / 2}, \\
\tau_{\infty}^{(0)}(0)=\frac{2 r_{g}}{3}\left(\frac{R_{b}^{3 / 2}}{r_{g}^{3 / 2}}-\frac{3^{3 / 2}}{2^{3 / 2}}\right), \tau_{\infty}^{(0)}\left(R_{b}\right)=\frac{2 r_{g}}{3}\left(\frac{R_{b}^{3 / 2}}{r_{g}^{3 / 2}}-1\right) .
\end{gathered}
$$

First of all, the center freezes at the moment $\tau_{\infty}^{(0)}(0)$, and the surface freezes later than all layers at $\tau_{\infty}^{(0)}\left(R_{b}\right)$ and the proper time interval of freezing of all layers is:

$$
\tau_{\infty}^{(0)}\left(R_{b}\right)-\tau_{\infty}^{(0)}(0)=\frac{2}{3}\left(\frac{3^{3 / 2}}{2^{3 / 2}}-1\right) r_{g} \approx 0.558 r_{g} .
$$

b) Elliptical and hyperbolic velocities $(k= \pm 1)$.

Plots of worldlines $r(R, t)$ for hyperbolic and elliptic solutions (55)-(56) are shown in Fig. 5-6. They show that when $t \rightarrow \infty$ the worldlines of particles in different layers $R$, as in the parabolic case, also tend to their asymptotes $r_{\infty}^{(k)}(R)$. We now find expressions for these asymptotes.

In (55)-(56), at $t \gg r_{g}$ we have $r_{ \pm} \rightarrow r_{g}$ and the logarithmic terms dominate. Therefore, equating the value of $r_{k}(R, r)$ in (51) to $r_{g}$ and substituting $r \rightarrow r_{\infty}^{(k)}(R)$, we obtain:

$$
r_{k}(R, r) \rightarrow-\frac{R_{b}}{k}\left(\frac{\left(1-k r_{g, R} / R\right)^{1 / 2}\left(1-k r_{\infty}^{(k)}(R) / R\right)}{\left(1-k r_{g} / R_{b}\right)^{1 / 2}}-1\right)=r_{g} .
$$

Expressing from this $r_{\infty}^{(k)}(R)$ gives a general formula for asymptotes of the inner layers:

$$
r_{\infty}^{(k)}(R)=\frac{R}{k}\left(1-\frac{\left(1-k r_{g} / R_{b}\right)^{3 / 2}}{\left(1-k r_{g} R^{2} / R_{b}^{3}\right)^{1 / 2}}\right) .
$$

In the limit $R_{b} \gg r_{g}$ or $k \rightarrow 0$, this formula reduces to (84). 
The same result also follows from the vanishing condition of the metric's time component $\exp \left[v\left(r_{\infty}\right)\right]=0$, which means the freezing of proper times:

$$
\frac{\left(1-k r_{g} / R\right)^{3 / 2}}{\left(1-k r_{g, R} / R\right)^{1 / 2}}-\left(1-k \frac{r_{\infty}^{(k)}}{R}\right)=0 .
$$

The inner asymptotes of the star, parallel to the center's worldline, are located almost equidistantly, by condensing near the surface only.

We find the freezing moments of the proper times of the layers $\tau_{\infty}^{(k)}(R)$ by substituting $r_{\infty}^{(k)}(R)$ into (26)-(27). For elliptical velocity $k=+1$, the freezing moments of the layers, as well as the center $\tau_{\infty}^{(+1)}(0)$ and surface $\tau_{\infty}^{(+1)}\left(R_{b}\right)>\tau_{\infty}^{(+1)}(0)$ are equal to:

$$
\begin{gathered}
\tau_{\infty}^{(+1)}(R)=\frac{R_{b}^{3 / 2}}{2 r_{g}^{1 / 2}}\left[\arccos \left(1-\frac{2 a_{+}^{3}}{b_{+}}\right)+2\left(1-\frac{a_{+}^{3}}{b_{+}}\right)^{1 / 2} \frac{a_{+}^{3 / 2}}{b_{+}^{1 / 2}}\right], \\
\tau_{\infty}^{(+1)}(0)=\frac{R_{b}^{3 / 2}}{2 r_{g}^{1 / 2}}\left[\arccos \left(1-2 a_{+}^{3}\right)+2\left(1-a_{+}^{3}\right)^{1 / 2} a_{+}^{3 / 2}\right], \\
\tau_{\infty}^{(+1)}\left(R_{b}\right)=\frac{R_{b}^{3 / 2}}{2 r_{g}^{1 / 2}} \arccos \left(\frac{2 r_{g}}{R_{b}}-1\right)+\frac{R_{b}^{2}}{r_{g}}\left(1-\frac{r_{g}}{R_{b}}\right)^{1 / 2} .
\end{gathered}
$$

For $k=-1$ the corresponding expressions have the form:

$$
\begin{gathered}
\tau_{\infty}^{(-1)}(R)=\frac{R_{b}^{3 / 2}}{r_{g}^{1 / 2}}\left\{2^{1 / 2}-\left[\frac{a_{-}^{3}}{b_{-}}\left(\frac{a_{-}^{3}}{b_{-}}-1\right)\right]^{1 / 2}+\ln \left(\left(\frac{a_{-}^{3}}{b_{-}}-1\right)^{1 / 2}+\frac{a_{-}^{3 / 2}}{b_{-}^{1 / 2}}\right)-\ln \left(1+2^{1 / 2}\right)\right\}, \\
\tau_{\infty}^{(-1)}(0)=\frac{R_{b}^{3 / 2}}{r_{g}^{1 / 2}}\left[2^{1 / 2}-\left[a_{-}^{3}\left(a_{-}^{3}-1\right)\right]^{1 / 2}+\ln \left(\frac{\left(a_{-}^{3}-1\right)^{1 / 2}+a_{-}^{3 / 2}}{1+2^{1 / 2}}\right)\right], \\
\tau_{\infty}^{(-1)}\left(R_{b}\right)=\frac{R_{b}^{3 / 2}}{r_{g}^{1 / 2}}\left[2^{1 / 2}-\left[\left(1+r_{g} / R_{b}\right) r_{g} / R_{b}\right]^{1 / 2} \ln \left(\frac{\left(1+r_{g} / R_{b}\right)^{1 / 2}+r_{g}^{1 / 2} / R_{b}^{1 / 2}}{1+2^{1 / 2}}\right)\right] .
\end{gathered}
$$

Let's find the asymptotic behavior of the dependence on $t$ the main variables at $t \gg r_{g}$, when $r_{k} \rightarrow r_{g}$. Eqs. (52)-(53) are simplified and worldlines take the form:

$$
r_{k} \simeq r_{g}\left[1+b_{k}(R) e^{-t / r_{g}}\right]
$$

where 


$$
\begin{aligned}
& b_{+1}=4\left(1-r_{g} / R_{b}\right) \exp \left[\frac{R_{b}}{r_{g}}-1+\left(R_{b}+2 r_{g}\right)\left(\frac{R_{b}}{r_{g}}-1\right)^{1 / 2} \arccos \frac{r_{g}^{1 / 2}}{R_{b}^{1 / 2}}\right], \\
& b_{-1}=2\left(1+r_{g} / R_{b}\right) \exp \left(1+r_{g} / R_{b}\right) \times \\
& \times \exp \left[\left(2-\frac{R_{b}}{r_{g}}\right)\left(2^{1 / 2}+\ln \frac{r_{g}^{1 / 2}+\left(R_{b}+r_{g}\right)^{1 / 2}}{R_{b}^{1 / 2}}\right)+\left(1+\frac{R_{b}}{r_{g}}\right)^{1 / 2}\right] .
\end{aligned}
$$

Substituting $r_{k}$ from (98) instead of $r$ in (51) we find the asymptotic behavior of worldlines:

$$
r_{k}(R, t) \simeq \frac{R}{k}\left[1-\frac{\left(1-k r_{g} / R_{b}\right)^{1 / 2}}{\left(1-k r_{g, R} / R\right)^{1 / 2}}\left(1-\frac{k r_{g}}{R_{b}}\left(1+b_{k} e^{-t / r_{g}}\right)\right)\right] .
$$

At $t \rightarrow \infty$ the Eq. (100) turns to (90). By inserting (100) into (26)-(27) we can determine the asymptotics of the proper time of the dust star's particles $\tau(t, R)$.

\subsection{Internal metric and its asymptotics}

\section{a. Parabolic velocity}

At $t \gg r_{g}$ the radial component of the metric in (61), taking into account (83), has the form:

$$
e^{\lambda(t, R)} \simeq \frac{1-R^{2} / 3 R_{b}^{2}+2 a /\left(3 e^{t / r_{g}}\right)}{1-R^{2} / R_{b}^{2}+2 a /\left(3 e^{t / r_{g}}\right)}, \quad e^{\lambda(\infty, R)} \simeq \frac{1-R^{2} / 3 R_{b}^{2}}{1-R^{2} / R_{b}^{2}} .
$$

In the center $R=0$ and on the surface $R=R_{b}$, the asymptotic values of $e^{\lambda(t, R)}$ are equal to $e^{\lambda(t, 0)}=1, e^{\lambda\left(t, R_{b}\right)} \simeq e^{t / r_{g}} / a$.

The temporary component at $t \gg r_{g}$, taking into account $y_{(0)} \simeq 1+a e^{-t / r_{g}}$, has the form [1]:

$$
e^{v(t, R)} \simeq e^{-2 t / r_{g}} \frac{3 a^{2}\left(1-R^{2} / 3 R_{b}^{2}\right)^{2}}{2\left[1-R^{2} / R_{b}^{2}+2 a /\left(3 e^{t / r_{g}}\right)\right]} .
$$

Its values in the center and on the surface: $e^{\nu(t, 0)} \simeq 3 a^{2} e^{-2 t / r_{g}} / 2$ and $e^{v\left(t, R_{b}\right)} \simeq a e^{-t / r_{g}}$. At $t \rightarrow \infty$ the metric (102) tends to:

$$
e^{v(\infty, R)}=e^{-2 t / r_{g}} \frac{3 a^{2}\left(1-R^{2} / 3 R_{b}^{2}\right)^{2}}{2\left(1-R^{2} / R_{b}^{2}\right)} .
$$

Thus, the freezing is stronger at the center than at the any other layer. 
2. Complete solution for a collapsing dust star

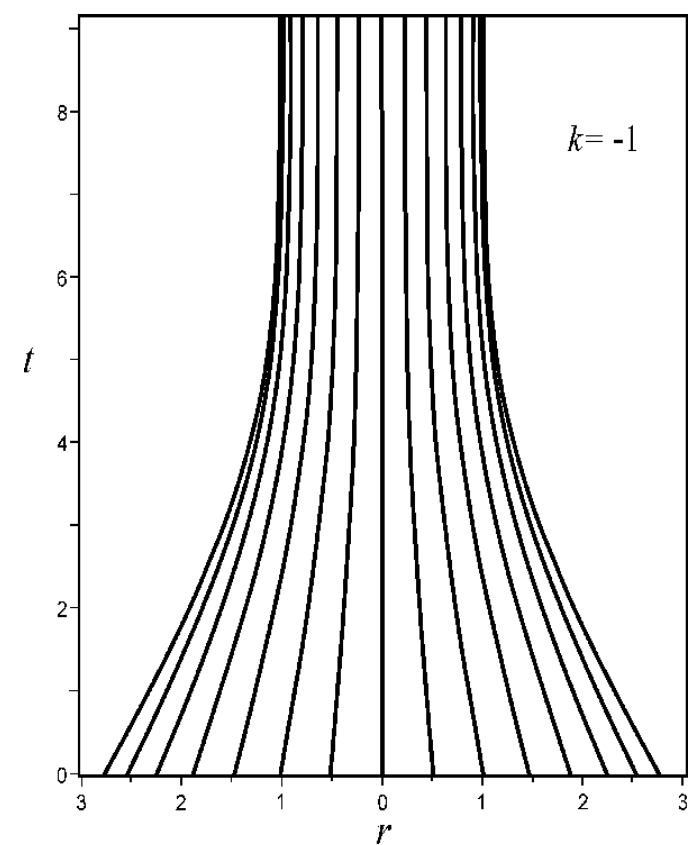

FIG. 5. The OC model worldlines of the dust star's particles along the star's diameter at the hyperbolic velocity ( $k=-1$ ) and $R_{b}=3.5$ at $\tau=0$ (in units $r_{g}$ ) according Eq. . At large $t$ the worldlines remain timelike, the surface freezes outside the gravitational radius, the internal layers freeze near own asymptotes given by Eq.

\section{b. Hyperbolic and elliptic velocities}

For large $t$, the radial metric in (61), after insertion $r_{k}$ from (100), takes the form:

$$
e^{-\lambda}=1+\frac{r_{g, R}}{k R}\left[\frac{\left(1-k r_{g} / R_{b}\right)^{1 / 2}}{\left(1-k r_{g, R} / R\right)^{1 / 2}}\left(1-k \frac{r_{g}}{R_{b}}\left(1+b_{k} e^{-t / r_{g}}\right)\right)-1\right]^{-1} .
$$

In the center and on the surface its values are equal to:

$$
e^{\lambda(t, 0)}=1, e^{\lambda\left(t, R_{b}\right)} \simeq a^{-1} e^{t / r_{g}} .
$$

At $t \rightarrow \infty$ the radial metric tends to its asymptote:

$$
e^{-\lambda(R, \infty)}=1+\frac{r_{g, R}}{k R}\left[\frac{\left(1-k r_{g} / R_{b}\right)^{1 / 2}}{\left(1-k r_{g, R} / R\right)^{1 / 2}}\left(1-k \frac{r_{g}}{R_{b}}\right)-1\right]^{-1} .
$$

The asymptotic behavior of the metric's time component (62) is found by substituting $r_{k}$ from (100) instead of $r$, which gives: 


$$
\begin{aligned}
& e^{v}=\frac{-R_{b} b_{k}^{2} e^{-2 t / r_{g}}}{k r_{g}\left(1-k r_{g} / R_{b}\right)^{1 / 2}} \times \\
& \times \frac{\left[\left(1-k r_{g} / R_{b}\right)^{3 / 2}-\left(1-k r_{g, R} / R\right)^{1 / 2}\right]^{2}}{\left[1-k\left(1+b_{k} e^{-t / r_{g}}\right) r_{g} / R_{b}\right]\left(1-k r_{g} / R_{b}\right)^{1 / 2}-k\left(1-k r_{g, R} / R\right)^{3 / 2}} .
\end{aligned}
$$

In the center and on the surface its values are equal to:

$$
e^{\nu(0)}=\frac{1-\left(1-k r_{g} / R_{b}\right)^{3 / 2}}{k r_{g}\left(1-k r_{g} / R_{b}\right)^{1 / 2}} R_{b} b_{k}^{2} e^{-2 t / r_{g}}, e^{\nu_{b}} \simeq b_{k} e^{-t / r_{g}} \simeq 1-r_{g} / r_{b} .
$$

\section{Conclusion}

The worldline of the surface at $r_{b}>r_{g}$ (and $\varphi, \theta=$ const.) is described by $t\left(R_{b}, r\right)$ and $\tau\left(R_{b}, r\right)$, denoting the same event of the same particle on the surface. There is a one-to-one correspondence between both curves $t(R, r)$ and $\tau(R, r)$, representing the same events on the same worldline, and therefore both curves are asymptotic. At $t \rightarrow \infty$ they asymptotically approach the gravitational radius $r(R, t) \rightarrow r_{g}, r(R, \tau(R, t)) \rightarrow r_{g}$.

Plots of worldlines of star particles (Fig. 3-5) clearly show the internal structure of a dust star on hypersurfaces of simultaneity. At large $t$ worldlines of particles in all layers approach their internal effective gravitational radii, which play the role of asymptotes parallel to the axis $t$ and located almost equidistant from the center, condensing only near the surface.

This shows that the picture of a frozen star refers not only to the surface asymptotically approaching the gravitational radius, always remaining outside it, but also to the structure of the dust star as a whole. A picture of the structure and evolution of a star with asymptotically frozen layers on the hypersurface of simultaneity will be reproduced in all reference frames from any of their coordinates if the correct correspondence with the external solution is ensured.

The transformations that are correct from the point of view of the general theory of relativity and astrophysics are those that describe at any finite moment $t$ only those events in the inner layers of the star that coincide with the moment $t$ on the surface. All other descriptions, based on non-simultaneous events in different parts of the star, are either nonphysical or will be auxiliary to the transition to a set of simultaneous events in the entire volume of the star.

It is also important that the worldlines of star particles in exact solutions of Einstein's equations cover every moment of the existence of these particles in the real world, and, therefore, only these solutions give us a complete picture of the evolution of the star. The irreversible slowdown of one's proper time relatively due to relativistic and gravitational slowdowns is the objective physical phenomenon that stops all processes in the star, including the collapse process itself. This fundamental physical phenomenon fundamentally distinguishes the collapse scenario in GR from the Newtonian one, where there is no such stopping mechanism. 
Thus, the collapse of a homogeneous dust star in GR, according to exact solutions at any finite moment of world time, leads to a frozen star or frozar, an object with an almost uniform and almost frozen internal structure with timelike worldlines of its particles.

\section{Appendix}

\section{Derivation of an expression for the auxiliary function $y$}

The diagonality condition (47) ( $y$ is written without index $k$ ):

$$
\frac{y^{\prime}}{\dot{y}}=\frac{\dot{r} r^{\prime}}{1-k r_{g, R} / R}=-\frac{\left(r_{g, R} r\right)^{1 / 2}}{R-k r_{g, R}}(1-k r / R)^{1 / 2}
$$

consider as an equation for $y$ and a solution we search among the functions having the form $[1,7]$ :

$$
y(R, r)=A(R)+B(R, r),
$$

where the independent on $r$ additive part of $y$ is denoted as $A$. For $B$, in the general case, the choice is too large and, therefore, we will search a particular solution, restricting ourselves to functions, when the total derivative of $B$ on $R$ disappears. Then we get:

$$
B^{\prime}=-\frac{\partial B}{\partial r} r^{\prime}, \quad y^{\prime}=A^{\prime}+B^{\prime}+\frac{\partial B}{\partial r} r^{\prime}=A^{\prime}, \quad \dot{y}=\frac{\partial B}{\partial r} \dot{r} .
$$

The Eq. (109) in this case takes the form:

$$
\frac{y^{\prime}}{\dot{y}}=A^{\prime}\left(\frac{\partial B}{\partial r} \dot{r}\right)^{-1}=\frac{\dot{r} r^{\prime}}{1-k r_{g, R} / R},
$$

which gives the equation for $A$ :

$$
A^{\prime}=\frac{\dot{r}^{2} r^{\prime}}{1-k r_{g, R} / R} \frac{\partial B}{\partial r}=\frac{r_{g, R}}{R-k r_{g, R}}\left[\frac{\partial B}{\partial r}\left(1-\frac{k r}{R}\right)\right]=\frac{r_{g, R}}{R-k r_{g, R}} q(R) .
$$

Here, the expression in square brackets is indicated as $q(R)$, since $A^{\prime}$ is independent on $r$ and, therefore, the right-hand side of (113) is also independent on $r$. This allows us to express $B$ as:

$$
\frac{\partial B}{\partial r}\left(1-\frac{k r}{R}\right)=q(R), \quad B=q \int \frac{d r}{1-k r / R}=-\frac{q R}{k} \ln (1-k r / R) .
$$

Hence, taking into account (111) and $r^{\prime}=r / R$, we obtain:

$$
\frac{q^{\prime}}{q}=-\frac{1}{R}, \quad \ln q=-\ln R+\ln w, \quad q=\frac{w}{R} .
$$

Thus, from (114) and (115) we arrive at the expression for $B$ :

$$
B=-\frac{w}{k} \ln (1-k r / R) .
$$

Substituting (115) into (113) allows us to find $A$ : 


$$
A^{\prime}=\frac{r_{g, R}}{R-k r_{g, R}} \frac{w}{R}=w \frac{R r_{g} / R_{b}^{3}}{1-R^{2} k r_{g} / R_{b}^{3}}, \quad A=-\frac{w}{2 k} \ln \frac{1-k r_{g, R} / R}{1-k r_{g} / R_{b}} .
$$

And finally, substituting (116)-(117) into (112), we find the final solution for $y_{(k)}$ :

$$
y_{(k)}(R, r)=-\frac{w}{k} \ln \frac{\left(1-k r_{g, R} / R\right)^{1 / 2}(1-k r / R)}{\left(1-k r_{g} / R_{b}\right)^{1 / 2}} .
$$

Derivatives of $y$, taking into account (25), are equal to:

$$
y^{\prime}=A^{\prime}=\frac{r_{g, R}}{R-k r_{g, R}} \frac{w}{R}, \quad \dot{y}=\frac{\partial B}{\partial r} \dot{r}=\frac{w \dot{r}}{R-k r}=-\frac{w r_{g, R}^{1 / 2}}{R r^{1 / 2}} \cdot \frac{1}{(1-k r / R)^{1 / 2}} .
$$

For $k= \pm 1$ the function $y_{(k)}$ is shown in (118), while for $k=0$ we find $y_{(0)}$ from (118) in the limit $k \rightarrow 0$ :

$$
y_{(0)}(R, r)=\frac{w r_{g}}{R_{b}}\left[\frac{1}{2}\left(\frac{R^{2}}{R_{b}^{2}}-1\right)+\frac{r R_{b}}{R r_{g}}\right] .
$$

Equation (112) determines $y_{(k)}$ only up to a constant $w$, which is present in (118). The solution for $y_{(0)}$ in the OS paper corresponds to the choice $w=R_{b} / r_{g}$ in (120). In the general case we find $w$ from the matching on the surface. It is clear that on the surface $t=M\left(r / r_{g}\right)$ and $y=r / r_{g}$, which, taking into account (110), (116) and (115), gives:

$$
y\left(R_{b}, r\right)=\frac{w}{R_{b}} r=\frac{r}{r_{g}}, \quad w=\frac{R_{b}}{r_{g}}, \quad q\left(R_{b}\right)=\frac{w}{R_{b}}=\frac{1}{r_{g}} .
$$

Hence we have:

$$
q=\frac{w}{R}=\frac{R_{b}}{R r_{g}}, \quad B=-\frac{R_{b}}{k r_{g}} \ln (1-k r / R) .
$$

Substituting this into (117), we obtain:

$$
A^{\prime}=\frac{R}{R_{b}^{2}} \frac{1}{1-k r_{g, R} / R}, \quad A=-\frac{R_{b}}{2 k r_{g}} \ln \frac{1-k r_{g, R} / R}{1-k r_{g} / R_{b}} .
$$

Thus, substituting (122)-(123) into (110) we find the final solution:

$$
y_{(k)}(R, \tau)=-\frac{R_{b}}{k r_{g}} \ln \frac{\left(1-k r_{g, R} / R\right)^{1 / 2}(1-k r / R)}{\left(1-k r_{g} / R_{b}\right)^{1 / 2}} .
$$

The derivatives of $y$, therefore, are equal to:

$$
y^{\prime}=A^{\prime}=\frac{R^{2}}{R_{b}^{2}} \frac{1}{R-k r_{g, R}}, \quad \dot{y}=-\frac{R^{2}}{R_{b}^{2}\left(r_{g, R} r\right)^{1 / 2}} \frac{1}{(1-k r / R)^{1 / 2}},
$$

and the diagonality condition (112) satisfies. 


\section{References}

1. Zakir Z. (2020) Quant. and Grav. Phys., 1:006-7132.

2. Oppenheimer J., Snyder H. (1939) Phys. Rev., 56, 455.

3. Friedmann, A. (1922). Z. Phys., 10, 376.

4. Tolman R. (1934) Proc. N. Acad. Sci . USA, 20, 169.

5. Klein, O. (1961). Ein. Prob. allgem Rel. в W. Heisenb. Phys. unz. Z., 58.

6. Weinberg S. (1972). Gravitation and Cosmology. Wiley.

7. Zakir Z. (2017) TPAC, 12(1) 1; 12(1) 17.

8. Zakir Z. (2018) Astroph. \& Space Sci. 363: 30.

9. Zakir Z. (2020) Theory of Frozars. Relativistic Collapse of Stars. CTPA, T.

10. Landau L. D., Lifshitz E. M. (1987) The Classical Theory of Fields. B. H. 